\title{
Difficulties in the diagnosis and treatment of malignant paraganglioma of the urinary bladder
}

\author{
Ewelina Rzepka' , Aleksandra Gilis-Januszewska ${ }^{1}$, Marta Opalińska ${ }^{1,2}$, Anna Sowa-Staszczak ${ }^{1,2}$, \\ Alicja Hubalewska-Dydejczyk ${ }^{1,2}$
}

${ }^{1}$ Clinical Department of Endocrinology, University Hospital, Jagiellonian University, Medical College, Krakow, Poland ${ }^{2}$ Nuclear Medicine Department, University Hospital, Jagiellonian University, Medical College, Krakow, Poland

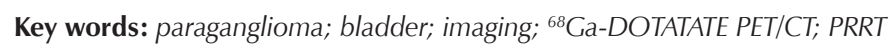

We present a history of 76-year-old female with haematuria, hypertension, headaches, and excessive sweating. Urine cytology revealed atypical cells. Contrast-enhanced CT showed a $4 \mathrm{~cm}$ lesion of the urinary bladder. MRI and subsequent cystoscopy confirmed the presence of a tumour on the anterior wall of the urinary bladder. Endoscopic partial cystectomy was performed. Histopathological examination confirmed paraganglioma (positive staining for neuroendocrine neoplasm markers, negative staining for keratins, Ki-67 -15-20\%). Repeated radical surgery (cystectomy) was not performed because of the patient's refusal; she was lost for further observation and management for several months. One year after initial diagnosis, the patient was referred to the Endocrinology Department. A 24-hour urine collection of metoxycatecholamines showed significantly elevated level of normetanephrine and 3-metoxythyramine (Tab. 1). Alpha-blocker treatment was initiated. Genetic testing for the major pheochromocytoma/paraganglioma (PCC/PGL) susceptibility genes (SDH-B, -D, VHL, and RET) was negative. Control CT scan of the abdomen and pelvis identified, apart from primary tumour, suspicious paraaortic and right iliac lymph nodes. ${ }^{131}$ I-metaiodobenzylguanidine (MIBG) SPECT/CT showed no tracer uptake (Fig. 1), whereas ${ }^{68} \mathrm{Ga}$-DOTATATE PET/CT identified pathological (Kerning score 4) somatostatin receptor expression in urinary bladder mass, thoracic, abdominal, and pelvic lymph nodes (Fig. 2). Based on those results, the diagnosis of malignant, disseminated paraganglioma was made.

The patient, who still refused any kind of surgery, was referred to peptide receptor radionuclide therapy (PRRT) with the use of ${ }^{177}$ Luthetium (Lu)-DOTATATE. The treatment tolerance was good without clinically relevant adverse events. However, follow-up CT scan done after
Table 1. Results of 24-hour urine collection of metoxycatecholamines

\begin{tabular}{lc}
\hline Normetanephrine & $3311.5 \mathrm{ug} / 24 \mathrm{~h}$ (URL $440 \mathrm{ug} / 24 \mathrm{~h})$ \\
\hline 3-metoxytyramine & $1580 \mathrm{ug} / 24 \mathrm{~h}$ (URL $220 \mathrm{ug} / 24 \mathrm{~h})$ \\
\hline Metanephrine & $62.7 \mathrm{ug} / 24 \mathrm{~h}$ (URL $341 \mathrm{ug} / 24 \mathrm{~h})$ \\
\hline URL - upper reference limit &
\end{tabular}

URL — upper reference limit

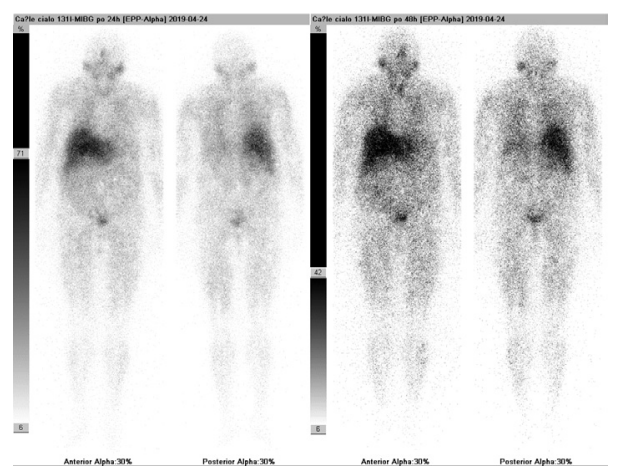

Figure 1. ${ }^{131}$ I-metaiodobenzylguanidine ( ${ }^{131} I$ MIBG) SPECT/ /CT - no pathological tracer uptake

the third of the planned four treatment cycles showed the disease progression, resulting in cessation of the treatment (Fig. 3). Because of very rapid progression of the disease, one of the pathological, supraclavicular lymph nodes was excised for histopathological assessment - the results showed progression of $\mathrm{Ki}-67$ to $42 \%$. At the time of reporting, the patient has remained in good clinical condition and she has been qualified for chemotherapy.

Paragangliomas (PGL) are rare tumours of neuroendocrine origin. Those localised in urinary bladder account for $10 \%$ of all PGLs. Approximately $10 \%$ of them are malignant. The common symptoms of the disease are haema-

Assoc. Prof. Aleksandra Gilis-Januszewska. MD, PhD, Clinical Department of Endocrinology, Jagiellonian University, Medical College,

University Hospital, Kopernika 17 St., 31-501 Krakow, Poland, tel/fax: (+48) 1242475 20/12 42473 99;

e-mail: myjanusz@cyf-kr.edu.pl 


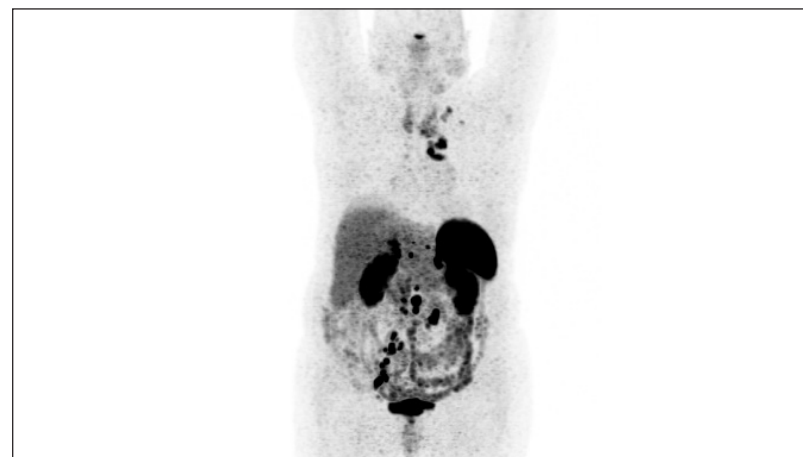

Figure 2. ${ }^{68} \mathrm{Ga}$-DOTATATE — pathological somatostatin receptor expression in thoracic, abdominal, and pelvic lymph nodes (arrows)

turia, which can be accompanied by hypertension, headache, palpitation, and micturition syncope, as the result of oversecretion of catecholamines [1]. Unrevealed hormonal activity of paragangliomas, without alpha-blockage, can jeopardise patient safety due to possible catecholamine crisis. The tumour catecholamine profile can provide information about possible germline mutations since there is a strong genotype - phenotype correlation in these neoplasms [2]. However, $50 \%$ of metastatic PGLs are sporadic [3]. Treatment options for metastatic PGLs are limited - apart from palliative surgery, they include radiotherapy, chemotherapy, and PRRT [1]. Generally, disease stabilisation and improvement of symptoms by the use of surgery and adjuvant therapies is observed in less than $50 \%$ of patients with metastatic disease [1].

The uptake of radiopharmaceuticals in metastatic PGLs depends on genetic background and differentiation status of the tumour. ${ }^{131} \mathrm{I}-\mathrm{MIBG}$ treatment is reserved for patients with relevant tumour avidity to ${ }^{123 / 131} \mathrm{I}-\mathrm{MIBG}$. Nonetheless, in metastatic PGLs ${ }^{123 / 131}$ I-MIBG tracer uptake is very often faint or even absent. Histopathologically, the lack of keratin expression in a presumed neuroendocrine neoplasm (NEN) should raise the suspicion of PGL [1, 2]. Histopathological distinction between PGL and NEN is very important because of the ability to overexpress somatostatin receptor 2 (SSR 2) presented by paragangliomas, which can lead to misdiagnosis when it is based on somatostatin receptor scintigraphy. Conversely, this ability enables 68Ga-DOTATATE PET/CT and PRRT use in the diagnosis and treatment of metastatic PGLs. Recent data of patients with sporadic and SDHB positive metastatic PGLs demonstrated the superiority of 68Ga-DOTATATE PET/CT in the detection of metastases over ${ }^{18} \mathrm{~F}$-FDG PET/CT, which is still recommended in the diagnosis of metastatic PGLs [2, 3].

Preliminary experience in the use of PRRT for surgically incurable PGLs suggests its low toxicity and favourable efficacy in disease control. However, progression

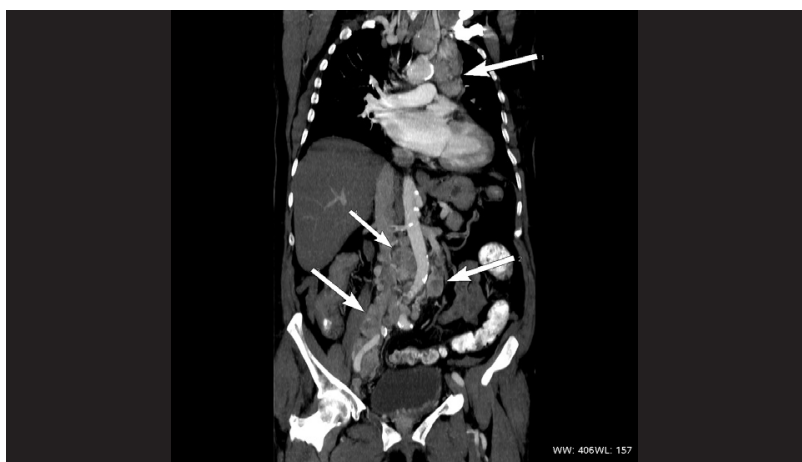

Figure 3. Follow-up computed tomography of the thorax, abdomen, and pelvis done after the third PRRT cycle revealed significant progression of paraaortic thoracic, abdominal, and pelvic lymph node metastases (arrows)

of the disease was noted in approximately $20 \%$ of the patients [4]. Lately, it has been shown that longitudinal increase in Ki-67 index and tumour grade was a common feature of pancreatic neuroendocrine tumours, and it was linked to a poor outcome [5]. We speculate that, in our case, transformation of metastases to higher grade (with doubled Ki-67 index compared to primary lesion), confirmed in histopathological examination of the lymph node, could be the reason for PRRT treatment failure regardless of very good somatostatin receptor expression on baseline tumour cells.

Nevertheless, further research is needed for a better understanding of the disease and response to the treatment.

\section{Author contributions}

The first authorship of E.R. and A.G.J. is of equal rank.

\section{Conflict of interest}

None declared.

\section{References}

1. Zhai H, Ma X, Nie W, et al. Paraganglioma of the Urinary Bladder: A Series of 22 Cases in a Single Center. Clin Genitourin Cancer. 2017; 15(5): e765-e771, doi: 10.1016/j.clgc.2017.03.010, indexed in Pubmed: 28688872.

2. Asa SL, Ezzat S, Mete O. The Diagnosis and Clinical Significance of Paragangliomas in Unusual Locations. J Clin Med. 2018; 7(9), doi: 10.3390/jcm7090280, indexed in Pubmed: 30217041.

3. Janssen I, Chen CC, Millo CM, et al. PET/CT comparing (68)Ga-DOTATATE and other radiopharmaceuticals and in comparison with CT/MRI for the localization of sporadic metastatic pheochromocytoma and paraganglioma. Eur J Nucl Med Mol Imaging. 2016; 43(10): 1784-1791, doi: 10.1007/s00259-016-3357-x, indexed in Pubmed: 26996779.

4. Satapathy S, Mittal BR, Bhansali A. 'Peptide receptor radionuclide therapy in the management of advanced pheochromocytoma and paraganglioma: A systematic review and meta-analysis'. Clin Endocrinol (Oxf). 2019; 91(6): 718-727, doi: 10.1111/cen.14106, indexed in Pubmed: 31569282.

5. Botling J, Lamarca A, Bajic D, et al. High-grade progression confers poor survival in pancreatic neuroendocrine tumors. Neuroendocrinology. 2019 [Epub ahead of print], doi: 10.1159/000504392, indexed in Pubmed: 31658459. 